\title{
AVALIAÇÃO DOS TEORES COMPOSTOS BIOATIVOS APÓS A SECAGEM DE RESÍDUO DE ACEROLA EM MICRO-ONDAS A VÁCUO
}

\author{
G. D. R.NOGUEIRA ${ }^{1 *}$,C. A. ALVARES ${ }^{1}$, K. M. CHANG ${ }^{1}$, C. R. DUARTE ${ }^{1}$, M. A. S. \\ BARROZO $^{1}$ \\ ${ }^{1}$ Universidade Federal de Uberlândia, Faculdade de Engenharia Química \\ e-mail: gdrnogueira@gmail.com
}

\begin{abstract}
RESUMO
A secagem é um processo indispensável para vários segmentos industriais. Hoje, destacam-se os estudos de métodos de secagem híbridos, ou seja, processos em que ocorre a associação de técnicas tradicionais e visam à obtenção de produtos de melhor qualidade e com menor gasto energético. A secagem realizada em micro-ondas a vácuo se apresenta como uma técnica híbrida promissora, pois combina rapidez, menores temperaturas e rápida transferência de energia das ondas eletromagnéticas, sendo uma tecnologia viável no reaproveitamento de resíduos agroindustriais. Neste trabalho foram avaliados os efeitos da potência das micro-ondas e o vácuo aplicado sobre os teores de compostos bioativos de resíduos de acerola. Valores para o teor de ácido ascórbico até seis vezes maiores do que o in natura foram encontrados, sendo o valor máximo de 1204, \pm mg ácido ascórbico/100 g de amostra seca. Flavonoides apresentaram um aumento de até três vezes quando o resíduo foi desidratado. Apesar de haver redução dos teores de fenólicos totais com a desidratação estes compostos ainda estão em níveis adequados para sua utilização em alimentos. O tempo de secagem, para todas as condições, reduziu quando comparado com técnicas tradicionais de secagem, como as convectivas. O resíduo de acerola seco pode ser uma boa fonte alimentar de compostos bioativos e a secagem a vácuo uma tecnologia promissora.
\end{abstract}

\section{INTRODUÇÃO}

A acerola (Malpighia glabra L.) é um fruto típico da América tropical, amplamente cultivada nas regiões Nordeste e Sudeste do Brasil. Os elevados teores de vitamina $\mathrm{C}$, ou ácido ascórbico, naturalmente encontrados neste fruto, têm favorecido a ampliação de oportunidades para o seu cultivo, processamento e comercialização (AGOSTINI-COSTA, ABEU e ROSSETTI, 2003).

O Brasil é o maior produtor, consumidor e exportador de acerola no mundo (CARVALHO et al., 2000), sendo processadas cerca de 34 mil toneladas de acerola por ano. Dessa forma, o Brasil também representa o maior produtor mundial de resíduos advindos do processamento industrial de acerola, sendo que estes representam entre 15 e $41 \%$ do volume total produzido (VASCONCELOS et al., 2002).

Após o processamento, o resíduo gerado é composto por uma mistura heterogênea de sementes, cascas e bagaço e são ricos em ácido ascórbico e compostos fenólicos e, geralmente, são decompostos para utilização como fertilizantes. Uma alternativa mais nobre para o reaproveitamento do resíduo agroindustrial seria a incorporação de farinhas de resíduos 
desidratados em novos alimentos (ABUD E NARIN, 2009). Farinhas do resíduo de acerola já foram incorporadas em cookies e barras de cereais (SILVA, 2015; MARQUES, 2013).

O ácido ascórbico está envolvido na síntese do colágeno, no desenvolvimento do tecido conjuntivo, no processo de cicatrização, na resistência a infecções, entre outras funções. É importante na resposta imune e em reações alérgicas (BASU, 2006).

Os fenólicos totais e flavonoides totais são compostos que agem como antioxidantes, não somente pela sua habilidade em doar hidrogênio ou elétrons, mas também em virtude de seus radicais intermediários estáveis, que impedem a oxidação de vários ingredientes do alimento, particularmente de lipídios. Eles podem ser pigmentos que dão a aparência colorida aos alimentos, ou produtos do metabolismo secundários, normalmente derivados de reações de defesa das plantas contra agressões do ambiente (BRANDWILLIAMS et al., 1995).

Vários autores já verificaram o aumento do teor de compostos bioativos, como fenólicos totais, flavonoides totais e o ácido ascórbico após a secagem (SILVA , 2015; SILVA, 2014; MARQUES ， 2013; BORTOLOTTI, 2012). Nóbrega (2012) constatou uma diminuição dos compostos bioativos após a secagem do resíduo de acerola comparado ao resíduo in natura. Entretanto o resíduo desidratado ainda foi considerado com elevado potencial bioativo.

$\mathrm{O}$ desenvolvimento da secagem de alimentos está relacionado com a demanda por produtos de mais fácil transporte, com menor volume e peso, armazenamento seguro e maior tempo de conservação (IBARZ e BARBOSACÁNOVAS, 2002). Na maior parte dos processos, a remoção de água se dá pelo contato do ar de secagem com o material a ser seco. Ainda há outros processos que não envolvem o fluxo de ar, como a liofilização, micro-ondas, por osmose e por meio de infravermelho (SEADER e HENLEY, 2006).

Destacam-se os estudos de métodos de secagem híbridos. As principais motivações no desenvolvimento de novas tecnologias de secagem híbrida são a minimização da degradação do produto final com a secagem até a umidade final desejada, além de possível redução de gasto enérgico durante o processo. (CHOU e CHUA, 2001).

$\mathrm{O}$ método de secagem envolvendo micro-ondas é distinto dos meios convencionais. As micro-ondas podem penetrar em materiais dielétricos e gerar um fluxo de calor interno (JIA, CLEMENT e JOLLY, 1993). Quando acoplado ao vácuo, a estrutura do material sofre expansão, o que facilita a liberação dos gases pelos poros (SAGAR e KUMAR, 2010). A redução da pressão ainda pode provocar a redução da temperatura e, assim melhorar os teores de compostos bioativos do produto final (JAYA e DAS, 2003).

Assim, este trabalho teve como objetivo avaliar a influência da potência das microondas e do vácuo, nos teores de fenólicos totais, flavonoides totais e ácido ascórbico do resíduo de acerola após a secagem.

\section{MATERIAIS E MÉTODOS}

\subsection{Material}

Os resíduos do processamento industrial da polpa de acerola foram cedidos pela empresa FrutPres da cidade de Presidente Olegário-MG. Os resíduos foram embalados em pacotes individuais de $100 \mathrm{~g}$, aproximadamente, envolvidos em papel alumínio, congelados e armazenados em freezer a $-18^{\circ} \mathrm{C}$.

\subsection{Secagem}

2.2.1 Equipamento 
Os testes de secagem foram realizados em um micro-ondas doméstico flat style da marca Panasonic com $800 \mathrm{~W}$ de potência e frequência de $2450 \mathrm{MHz}$. O forno micro-ondas foi adaptado para introduzir uma mangueira de silicone conectando um balão de fundo chato ao sistema de vácuo composto por um kitassato e uma bomba de vácuo marca Prismatec 2 VC.

\subsubsection{Potência Fornecida à Amostra}

Por calorimetria a potência nominal do aparelho de micro-ondas foi relacionada com a energia fornecida à amostra, como realizado por Cui et al. (2004), Rosa (2010) e Béttega et al. (2013).

Determinada variação de temperatura de uma massa de água conhecida, submetida às micro-ondas por um período de tempo, a Equação 1 foi então aplicada. Nela se assume que a variação de temperatura se deve somente à energia aplicada pelo magnetron, sendo a variação do calor específico com a temperatura considerada desprezível. Os testes foram feitos em duplicata antes e após as secagens, para garantir condições constantes nas potências programadas durante $o$ planejamento experimental.

$$
Q_{a b s}=\frac{m_{a} c_{p a} \Delta T}{t}
$$

\subsubsection{Condições de secagem}

A análise dos teores de compostos bioativos do resíduo de acerola foi realizada avaliando a influência de duas variáveis independentes: potência do micro-ondas (200, 280,480 e $600 \mathrm{~W}$ ) e o vácuo proporcionado pela bomba. As pressões utilizadas foram variáveis de 0 a $400 \mathrm{mmHg}$ abaixo da pressão atmosférica local, que em Uberlândia é, em média, $760 \mathrm{mmHg}$.

\subsubsection{Procedimento experimental}

Antes de cada experimento a amostra foi retirada do freezer e colocada para descongelar a $5^{\circ} \mathrm{C}$ por um período de $24 \mathrm{~h}$. Testes de umidade foram realizados em triplicata a fim de determinar a umidade inicial. Cerca de $60 \mathrm{~g}$ do resíduo de acerola foram, então, transferidas para o balão de fundo chato que foi posicionado dentro do forno micro-ondas e conectado ao sistema de vácuo.

Após ajustadas a pressão e a potência, o experimento foi iniciado, sendo a amostra pesada periodicamente $(5 \mathrm{~min}$ para as potências de 200 e $280 \mathrm{~W}$ e $2,5 \mathrm{~min}$ para potências de 480 e $600 \mathrm{~W}$ ). A massa foi relacionada com a umidade através da Equação 2 e os experimentos foram realizados até a umidade final de $10 \pm 1,5 \%(\mathrm{~m} / \mathrm{m}$, em base úmida). Este teor de umidade foi estabelecido a fim de evitar a queima do produto final.

$$
U=\frac{\left(m_{t}-m_{b}\right)-\left(m_{o}-m_{b}\right)\left(1-\frac{U_{o}}{100}\right)}{\left(m_{t}-m_{b}\right)} 100
$$

\subsection{Análises}

\subsubsection{Umidade}

A umidade do material foi determinada por meio do método da estufa. Neste método a amostra foi disposta em estufa a $105^{\circ} \mathrm{C}$ por $24 \mathrm{~h}$ e após esse período a amostra transferida para um dessecador até resfriar e ser pesada em balança analítica.

\subsubsection{Teor de Fenólicos Totais}

Foi utilizado o método de Folin-Ciocalteu (SINGLETON e ROSSI, 1965) para determinar o teor de fenólicos totais, sendo metanol utilizado como solvente extrator. A curva padrão com ácido gálico foi preparada com concentrações variáveis de 0,2 a 2,0 $\mathrm{mg} / \mathrm{mL}$ e lida em espectrofotômetro com comprimento de onda de $450 \mathrm{~nm}$. Os experimentos foram realizados em triplicata para duas massas distintas e os resultados 
expressos em mg de ácido gálico equivalente (AGE)/100 g de amostra, em base seca.

\subsubsection{Teor de Flavonoides Totais}

Para o teor de flavonoides totais o método colorimétrico de Zhishen et al. (1999) foi empregado. Como solvente extrator também foi utilizado o metanol, já o padrão foi utilizado a rutina e a leitura efetuada no comprimento de onda de $622 \mathrm{~nm}$.

Para cada experimento foram feitas análises em triplicata para duas massas distintas, totalizando assim seis resultados por condição. Todos os resultados foram expressos em $\mathrm{mg}$ de rutina/100 $\mathrm{g}$ de amostra em base seca.

\subsubsection{Teor de ácido ascórbico}

Foi utilizada a metodologia de Dürust et al. (1997) com adaptações. O espectrofotômetro foi zerado com água destilada e, no comprimento de onde de 520 nm, a absorbância da mistura das soluções de ácido oxálico $0,4 \%(0,5 \mathrm{~mL})$ com tampão de acetato $(0,5 \mathrm{~mL})$ e 2,6-diclorofenol-indofenol $0,05 \%$ (4 mL). Foram realizadas seis leituras e a média foi denominada de $\mathrm{L}_{1}$. Todas as leituras foram realizadas após $15 \mathrm{~s}$.

Para a curva padrão foram preparadas soluções de ácido ascórbico com concentrações variadas de 5 a $70 \mathrm{mg} / \mathrm{L}$. Foram realizadas em triplicatas leituras da absorbância das misturas de cada solução padrão de ácido ascórbico $(0,5 \mathrm{~mL})$ com tampão de acetato $(0,5 \mathrm{~mL})$ e 2,6-diclorofenolindofenol $0,05 \%$ (4 mL) e estas absorbâncias foram designadas como $\mathrm{L}_{2}$. A curva padrão foi obtida através da regressão linear da diferença $\mathrm{L}_{1}-\mathrm{L}_{2}$ com a concentração de ácido ascórbico.

As amostras foram trituradas e massas de $0,5 \mathrm{~g}$, aproximadamente, separadas para a análise. A seguir foram adicionados $25 \mathrm{~mL}$ de solução de ácido oxálico $(0,4 \%, \mathrm{~m} / \mathrm{v})$ a cada massa e a mistura agitada mecanicamente em tubos do tipo vortex. Esta mistura foi filtrada e o extrato obtido utilizado para as determinações de novos $\mathrm{L}_{2}$. $\mathrm{O}$ branco foi feito substituindo a solução de DCFI por água destilada. Em cada experimento foram feitas triplicatas em duas massas distintas, obtendo seis resultados. Os resultados foram expressos em mg ácido ascórbico (AA)/100 g de amostra (base seca).

\subsubsection{Tratamento Estatístico}

A quantificação dos efeitos das variáveis independentes nos teores de compostos bioativos foi realizada por meio de técnicas de regressão.

\section{RESULTADOS E DISCUSSÕES}

\subsection{Energia fornecida à amostra}

A Figura 1 mostra a relação entre a potência fornecida pelo equipamento à amostra e a potência nominal do equipamento, a regressão linear executada e $\mathrm{o}$ seu coeficiente de determinação $\left(\mathrm{R}^{2}\right)$. $\mathrm{O}$ desvio padrão médio para a potência absorvida em cada nível de potência variou de 0,47 a $4,13 \%$ e a curva obtida através da regressão linear apresentou um $\mathrm{R}^{2}$ de 99,35\%.

Figura 1- Energia absorvida pela água em função da potência

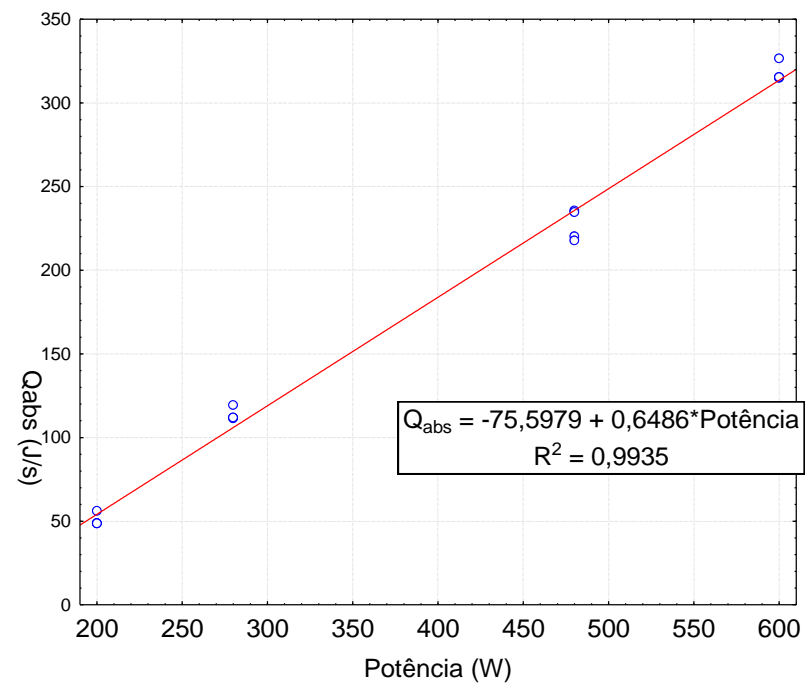


Nota-se, assim, que a potência fornecida pelo forno micro-ondas se manteve constante e o calor absorvido pela amostra é proporcional à potência aplicada. Resultados similares foram obtidos por Rosa (2010), ao avaliar a reprodutibilidade da energia fornecida por um equipamento semelhante.

Variações da potência fornecida pelo equipamento podem ocorrer, pois o magnetron pode envelhecer e seus filamentos necessitarem de mais tempo para atingir as condições de emissão, ou ainda devido a operação prolongada do forno micro-ondas aquecer o magnetron causando a redução do campo magnético (CUI et al., 2004). Portanto, medir a potência fornecida pelo equipamento periodicamente é essencial para garantir as condições operacionais constantes.

\subsection{Compostos Bioativos}

Foram avaliados neste trabalho os teores de ácido ascórbico, de fenólicos e flavonoides totais do resíduo de acerola seco em microondas a vácuo até a umidade final de $10 \pm 1,5 \%$ ( $\mathrm{m} / \mathrm{m}$, em b.u.). Por isso, o tempo de cada corrida foi variável. Na Tabela 1 são apresentados os teores dos compostos bioativos ao final de cada experimento e para o resíduo in natura, além dos tempos totais de secagem.

Tabela 1: Condições experimentais, médias dos teores de compostos bioativos com desvio padrão e tempo de secagem de cada experimento.

\begin{tabular}{ccccccc}
\hline $\begin{array}{c}\text { Experi- } \\
\text { mento }\end{array}$ & $\begin{array}{c}\text { Potência } \\
(\mathrm{W})\end{array}$ & $\begin{array}{c}\text { Vácuo } \\
\text { aplicado } \\
(\mathrm{mmHg})\end{array}$ & $\begin{array}{c}\text { Tempo } \\
\text { de } \\
\text { secagem } \\
(\mathrm{min})\end{array}$ & $\begin{array}{c}\text { Teor de Ácido } \\
\text { Ascórbico } \\
(\mathrm{mg} / 100 \mathrm{~g})\end{array}$ & $\begin{array}{c}\text { Teor de } \\
\text { Fenólicos } \\
\text { Totais } \\
(\mathrm{mg} / 100 \mathrm{~g})\end{array}$ & $\begin{array}{c}\text { Teor de } \\
\text { Flavonoides } \\
\text { Totais } \\
(\mathrm{mg} / 100 \mathrm{~g})\end{array}$ \\
\hline 1 & 280 & 100 & 25 & $820,58 \pm 16,63$ & $378,64 \pm 8,75$ & $3,57 \pm 0,88$ \\
2 & 480 & 100 & 12.5 & $958,56 \pm 32,90$ & $396,24 \pm 15,91$ & $3,72 \pm 0,13$ \\
3 & 280 & 300 & 29.5 & $1100,96 \pm 18,59$ & $403,02 \pm 11,26$ & $3,79 \pm 0,09$ \\
4 & 480 & 300 & 12 & $1204,10 \pm 35,21$ & $406,31 \pm 16,41$ & $4,27 \pm 0,21$ \\
5 & 280 & 200 & 25 & $1091,54 \pm 54,18$ & $410,49 \pm 38,78$ & $3,48 \pm 0,46$ \\
6 & 280 & 200 & 26.5 & $1077,81 \pm 39,51$ & $408,58 \pm 10,16$ & $3,45 \pm 0,23$ \\
7 & 200 & 200 & 82 & $1018,96 \pm 18,84$ & $414,99 \pm 7,89$ & $4,14 \pm 0,25$ \\
8 & 600 & 200 & 11.5 & $1201,24 \pm 45,38$ & $464,34 \pm 23,22$ & $4,43 \pm 0,55$ \\
9 & 280 & 400 & 27 & $1158,48 \pm 90,44$ & $404,55 \pm 42,05$ & $4,18 \pm 0,22$ \\
10 & 280 & 0 & 33 & $662,19 \pm 29,79$ & $335,93 \pm 2,59$ & $3,03 \pm 0,82$ \\
In natura & - & - & - & $193,82 \pm 10,11$ & $1493,97 \pm 33,51$ & $2,39 \pm 0,16$ \\
\hline
\end{tabular}

Através da Tabela 1, verifica-se que resíduo de acerola in natura apresenta teor de fenólicos totais de 1493,97mg AGE/100 g. Silva (2015) obteve 1352,42 mg AGE/100 g de resíduo de acerola in natura (base seca), valor similar ao obtido neste trabalho. Nóbrega (2012) e Oliveira (2009) encontraram valores de 2241,39 e $680 \mathrm{mg}$ AGE/100 g, respectivamente.

A amostra in natura apresenta uma alta concentração de compostos bioativos, pois este é superior a $1000 \mathrm{mg}$ AGE/100 g. Amostras com concentrações compreendidas entre 100 e $1000 \mathrm{mg}$ AGE/100 g são de valores intermediários e abaixo deste intervalo são 
consideradas baixas concentrações (VASCO, 2009).

Apesar da redução do teor de fenólicos totais após a secagem, estes teores continuam sendo considerados de valor intermediário, segundo a classificação mencionada acima. O valor mínimo obtido foi de $335,96 \mathrm{mg}$ AGE/100 g (base seca) na potência de $280 \mathrm{~W}$ e pressão atmosférica

O teor de flavonoides totais observado para o resíduo in natura foi de $2,39 \mathrm{mg}$ de rutina/100 g de resíduo (base seca), valor similar aos relatados por Silva (2015), Silva (2014) e Bortolotti (2012) que foram de 1,65, 1,0 e $0,80 \mathrm{mg}$ de rutina/100 $\mathrm{g}$ de resíduo (base seca). Cabe ainda ressaltar que grandes variações de compostos bioativos podem ser verificados na literatura, pois estes são dependentes da safra, local de cultivo e modo de processamento dos frutos.

Observa-se ainda na Tabela 1 que os teores de ácido ascórbico, assim como os teores de flavonoides totais, após a secagem foram superiores ao resíduo in natura .

A fim de quantificar o comportamento dos compostos bioativos em função das variáveis estudadas foram realizadas regressões múltiplas a partir dos resultados experimentais (Tabela 1).

A função obtida na regressão do teor de fenólicos totais (TPT) teve um $\mathrm{R}^{2}$ de $98,11 \%$ e é descrita pela Equação 3, em que $\mathrm{x}$ é a potência (W) e y o vácuo aplicado $(\mathrm{mmHg})$. $\mathrm{Na}$ regressão todos os fatores, assim como seus termos quadráticos e a interação entre eles foram significativos estatisticamente.

$$
\begin{aligned}
T P T=435,22 & -0,66 x+0,63 y \\
& +1,1 \cdot 10^{-2} x^{2} \\
& -7,8 \cdot 10^{-4} y^{2} \\
& -5,56 \cdot 10^{-4} x y
\end{aligned}
$$

Na Figura 2 é apresentada a superfície de resposta para o teor de fenólicos totais em função das variáveis analisadas. Pode-se verificar que em níveis de potência mais baixos, o vácuo aplicado exerce uma maior influência, aumentando o valor do teor de fenólicos totais com o aumento do vácuo aplicado. Para níveis mais altos de potência esse efeito não é mais tão significativo, tendo suas concentrações máximas em pressões intermediárias.

Figura 2 - Superfície para o teor de fenólicos totais em função do vácuo aplicado e da potência.

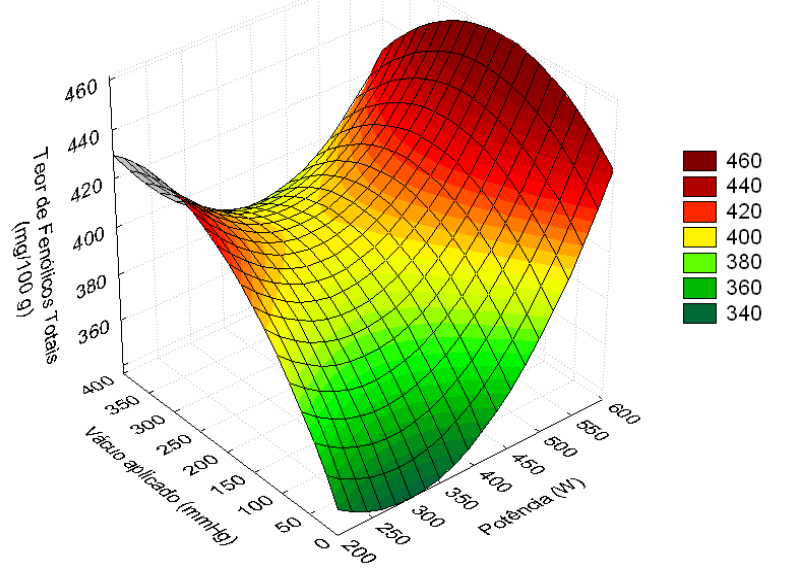

Wojdylo, Figiel e Oszmianski (2009), secando morangos em micro-ondas a vácuo, também encontraram teores de fenólicos inferiores ao do fruto in natura e com o mesmo comportamento qualitativo verificado neste trabalho em relação à potência. Sugerindo, assim, que as perdas de fenólicos se devem à degradação térmica com o aumento do calor e do tempo de secagem. O mesmo foi verificado por Calín-Sánchez et al. (2013) e Calín-Sánchez et al. (2014) para fatias de alho e romã quando secas em microondas a vácuo.

$\mathrm{Na}$ regressão múltipla para o teor de flavonoides totais (TFT), o termo quadrático do vácuo aplicado e o termo da interação entre vácuo e potência não foram estatisticamente significativos. A função possui um $\mathrm{R}^{2} \mathrm{de}$ $86,81 \%$ e é apresentada na Equação 4. A 
superfície de resposta é apresentada na Figura 3.

$$
\begin{aligned}
\text { TFT }=4,96- & 1,09 \cdot 10^{-2} x \\
& +2,56 \cdot 10^{-3} y \\
& +1 \cdot 5610^{-5} x^{2}
\end{aligned}
$$

Figura 3 - Superfície para o teor de flavonoides totais em função do vácuo aplicado e da potência.

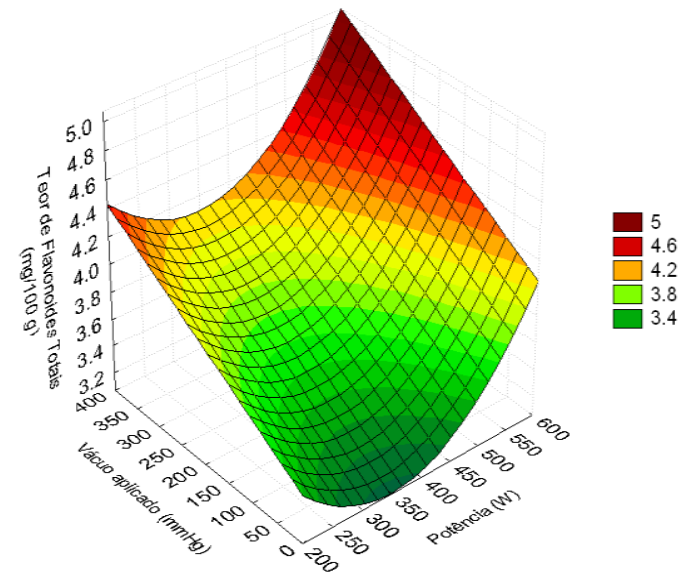

$\mathrm{Na}$ Figura 3, nota-se que o efeito positivo do vácuo aplicado e da potência no aumento do teor de flavonoides totais. Wojdylo et al. (2014) avaliaram a variação dos compostos fenólicos na secagem de Sour Cherries em micro-ondas a vácuo e constataram que o teor de compostos fenólicos, dentre eles os flavonoides, decaía com a redução da potência. Porém a influência do vácuo não foi avaliada.

Os fenólicos e flavonoides, como compostos do metabolismo secundário, tem concentração elevada nos vacúolos. Os quais se rompem com maior facilidade com o aumento da potência, liberando assim tais compostos (CHISM e HAARD, 1996).

Observa-se nas Figuras 2 e 3 que os teores de fenólicos e flavonoides totais são, ligeiramente, superiores quando a secagem é feita na potência de $200 \mathrm{~W}$ em relação aos teores após a secagem em potências intermediárias. Isto sugere que as temperaturas em baixas potências são moderadas e, apesar de dificultar a extração de compostos ligados, evitam sua degradação térmica (WOJDYLO et al., 2014).

A regressão para o teor de ácido ascórbico (TAA) apresentou um $\mathrm{R}^{2}$ de $96,58 \%$ e é descrita pela Equação 5. Nela o termo quadrático da potência e o termo da interação entre as variáveis não se mostraram significativos estatisticamente. Na Figura 4 é apresentada a superfície de resposta para o comportamento do o teor de ácido ascórbico.

$$
\begin{array}{r}
T A A=522,95+0.42 x+2,81 y \\
-3,85.10^{-3} y^{2}
\end{array}
$$

Figura 4 - Superfície para o teor de flavonoides totais em função do vácuo aplicado e da potência.

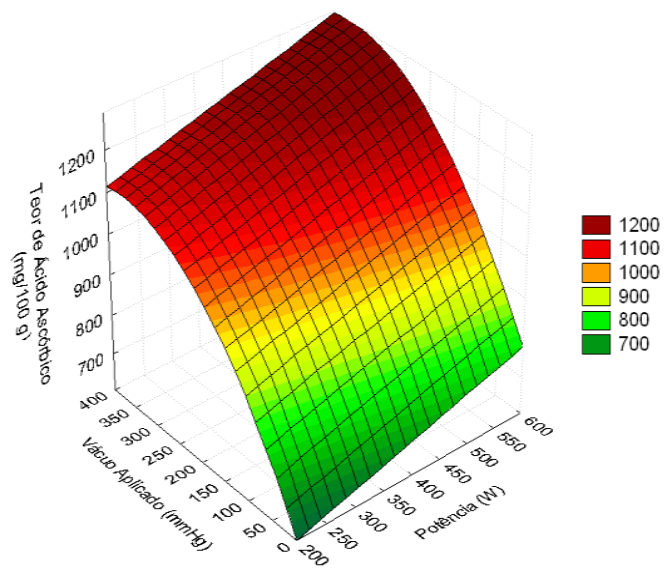

Pode-se observar que, na Figura 4, que o teor de ácido ascórbico é mais influenciado pelo vácuo aplicado que pela potência das micro-ondas. Após a secagem o resíduo passa a apresentar valores substancialmente superiores ao do resíduo in natura, como observado na Tabela 1.

O aumento do teor de ácido ascórbico pode estar ligado com a facilidade após a secagem de se extrair o composto. Após o 
processo, o composto que antes estava ligado é liberado devido ao rompimento de constituintes celulares durante a secagem (CHISM e HAARD, 1996).

O aumento da temperatura, que é proporcional à potência, também pode desativar enzimas que contribuíam para a degradação do ácido ascórbico (WOJDYLO, FIGIEL e OSZMIANSKI, 2009). O aumento do vácuo reduz a quantidade de oxigênio em contato com a amostra, o que também previne a degradação do ácido ascórbico por oxidação.

\section{CONCLUSÃO}

Neste trabalho, estudou-se comportamento do teor de fenólicos totais, de flavonoides totais e de ácido ascórbico após a secagem em micro-ondas a vácuo, quantificando o efeito da potência e do vácuo aplicado.

Os teores de compostos bioativos apresentam maiores concentrações quando a secagem ocorreu em potências mais altas. Isto foi devido às concentrações elevadas destes compostos em estruturas celulares que são rompidas com o processo de secagem.

A diminuição do oxigênio disponível, causada pela diminuição da pressão, também está relacionado com o aumento do teores de compostos bioativos. Pois há redução da degradação dos mesmos em reações de oxidação.

\section{NOMENCLATURA}

$\Delta T=$ Variação de temperatura $\left({ }^{\circ} \mathrm{C}\right)$

$c_{p a}=$ Calor específico da água $\left(\mathrm{J} / \mathrm{g}^{\circ} \mathrm{C}\right)$

$m_{a}=$ Massa de água $(\mathrm{g})$

$m_{t}=$ Massa do conjunto balão e amostra em um determinado momento $(\mathrm{g})$

$m_{b}=$ Massa do balão $(\mathrm{g})$

$m_{o}=$ Massa inicial do conjunto balão e amostra (g)

$Q_{a b s}=$ Energia absorvida pela massa de água $(\mathrm{J} / \mathrm{s})$

$t=$ Tempo $(\mathrm{s})$

$U=$ umidade da amostra em um determinado momento (\%)
$U_{o}=$ umidade inicial da amostra $(\%)$

\section{REFERÊNCIAS}

ABUD, A. K. S.; NARAIN, N. Incorporação da farinha de resíduo do processamento de polpa de fruta em biscoitos: uma alternativa de combate ao desperdício. Brazilian Journal of Food Technology, v.12, p.257-265, 2009.

AGOSTINI-COSTA, T. S.; ABREU, L N.; ROSSETTI, A. G. Efeito do congelamento e do tempo de estocagem de polpa de acerola sobre 0 teor de carotenóides, Revista Brasileira de Fruticultura, v. 25, p.56-58, 2003.

BASU, T. K.; DICKERSON, J. W. Vitamins in Human Health and Disease. $C A B$ International, 2006.

BÉTTEGA, R.; ROSA, J. G.; CORRÊA, R. G.; FREIRE, J. T. Comparison of carrot (Daucus carota L) Drying in Microwave and in vaccum microwave. Brazilian Journal of Chemical Engineering, v.31, p.403-412, 2014.

BORTOLOTTI, C. T. Estudo Experimental da Fluidodinâmica de Uma Mistura de Resíduo de Acerola e Sola em Leito de Jorro. 2012. 101p. Dissertação (Mestrado em Engenharia Química) - Universidade Federal de Uberlândia, Uberlândia, 2015

BRAND-WILLIAMS,W.; CUVELIER, M. E.; BERSET, C. Use of a free radical method to evaluate antioxidant activity. LebensmittelWissenschaft Technologie, London, v. 28, p. 25-30, 1995.

CALÍN-SÁNCHEZ， A., FIGIEL， A., HERNÁNDEZ, F., MELGAREJO, P., LECH, K., CARBONELLBARRACHINA, A.A. Chemical composition antioxidant capacity, and sensory quality of pomegranate 
(Punicagranatum L.) arils and rind as affected by drying method. Food Bioprocess Technology.V.6, p.1644-1654, 2013.

CALÍN-SÁNCHEZ, A.; FIGIEL, A.; WOJDYLO, A; SZARYCZ, M.; BARRACHINA, A. C. Drying of Garlic Slices Using Convective Pre-drying and Vacuum Microwave Finishing Drying: Kinetics, Energy Consumption, and Quality Studies. Food Bioprocess Technology, v.7, p.398 408, 2014.

CARVALHO, R. A.; FERREIRA, C. A. P.; NASCIMENTO Jr., J. D. B.; MENEZES, A. J. E. A.; SUZUKI, E.; SASAKI, G. Análise econômica da produção de acerola no município de Tomé-Açu, Belém: Embrapa Amazônia Oriental, 21p., 2000.

CHISM, G. W., \&HAARD, N. F. Characteristics of Edible Plant Tissues. In: O. R. Fennema (Ed.), Food chemistry(pp. 943-1011). New York, Marcel Dekker Inc., 1996.

CHOU, S. K. e CHUA, K. J.New hybrid drying Technologies for heat sensitive foodstuffs. Trends in Food Science \& Technology, v. 12, p.359-369, 2001.

CUI, Z. W.; XU, S. Y.; SUN, D. W. Microwave-Vaccum Drying Kinetics of Carrot Slices. Journal of Food Engineering, v. 65, p.157-164, 2004.

DÜRUST, N.; GOGAN, S.; DÜRUST, Y. Ascorbic Acid and Element Content of Foods of Trabzon (Turkey). Journal of Agricultural and Food Chemistry, v.45, p.2085-2087, 1997.

IBARZ, A.; BARBOSA-CANOVÁS, G. V. Unit Operations in Food Engeneering. Boca Raton: CRC Press; 2002.
JAYA S, DAS H. A vacuum drying model for mango pulp.Drying Technology, v.21, p.1215-1234, 2003.

JIA, X., CLEMENTS, S., \& JOLLY, P. Study of heat pump assisted microwave drying. Drying Technology, v.11, p.1583-1616, 1993.

MANACH, C.; SCALBERT, A.; MORAND, C.; RÉMÉSY, C.; JIMÉNEZ, L. Polyphenols: food sources and bioavailability. American Journal of Clinical Nutrition, v.79, p.727$747,2004$.

MARQUES, T. R. Aproveitamento Tecnológico de Resíduos De Acerola: Farinhas e Barras de Cereais. 2013. 101p. Dissertação (Mestrado em Agroquímica) Universidade Federal de Lavras, Lavras, 2013.1

NÓBREGA, E. M. M. A. Secagem do Resíduo de Acerola (Malpighia emarginata DC.): Estudo do Processo e Avaliação do Impacto sobre o Produto Final. 2012. 116p. Dissertação (Mestrado em Engenharia Química) - Universidade Federal do Rio Grande do Norte, Natal, 2012.

OLIVEIRA, A. C. Total phenolic content and free radical scavenging activities of methanolic extract powders of tropical fruit residues. Food Chemistry, v.115, p.469-475, 2009.

ROSA, J. G. Secagem de Cenoura (Daucuscarota L.) em Microondas. 2010. Dissertação (Mestrado em Engenharia Química) - Universidade Federal de São Carlos, São Carlos, 2010.

SAGAR VR, SURESH KUMAR P (2010) Recent advances in drying and dehydration of fruits and vegetables: a review. Journal Food 
Science and Technology, v,47, p.15-26, 2010.

SEADER, J. D.; HENLEY, E. J. Separation Process Principles. $2^{\mathrm{a}}$ edição. Danvers: John Wiley \& Sons Inc.; 2006

SILVA, D. S. Estudo da Transferência de Calor e Massa na Secagem em Leito Fixo Visando o Aproveitamento de Resíduo de Acerola (Malpighia emarginata DC.). 2015. 216p. Tese (Doutorado em Engenharia Química) - Universidade Federal de Uberlândia, Uberlândia, 2015.

SILVA, P. B. Secagem de Resíduos de Frutas em Secador Roto-Aerado. 2014. 93p. Dissertação (Mestrado em Engenharia Química) - Universidade Federal de Uberlândia, Uberlândia, 2015.

SINGLETON, V. L.; ROSSI, J.A. Jr. Colorimetry of total phenolics with phosphomolybdic-phosphotungstic acid reagents. American Journal Enology and Viticulture, v.16, p. 144-158, 1965.

TURNER, I. W.,\& JOLLY, P. Combined microwave and convective drying of a porous material. Drying Technology - An International Journal, v.9, p.1209-1270, 1991.

VASCO, C. Phenolic Compounds in Ecuadorian fruits.Tese (Doutorado em Ciência dos Alimentos) - Swedish University of Agricultural Sciences, Faculty of Natural Resources and Agricultural Sciences, Department of Food Science, Uppsala, 2009.

VASCONCELOS, V. R.; NEIVA, J. N. M.; PIMENTEL, J. C. M. Utilização de subprodutos do processamento de frutas na alimentação de caprinos e ovinos. In: VI Seminário Nordestino de Pecuária PECNORDESTE, 2002, Fortaleza. Anais... Fortaleza: FAEC, 2002, p.83-99.
WOJDYLO, A.; FIGIEL, A.; LETCH, K.; NOWICKA , P.; OSZMIANSKI, J. Effect of Convective and Vacuum-Microwave Drying on the Bioactive Compounds, Color, and Antioxidant Capacity of Sour Cherries. Food Bioprocess Technology, v.7, p.829-841, 2014.

WOJDYLO, A.; FIGIEL, A.; OSZMIANSKI, J. Effect of Drying Methods with the Application of Vacuum Microwaves on the Bioactive Compounds, Color, and Antioxidant Activity of Strawberry Fruits. Journal of Agricultural \&. Food Chemistry, v.57, p.1337-1343, 2009.

WOLFE, K.; WU, X.; LIU, R. H. Antioxidant activity of apple peels. Journal of Agricultural and Food Chemistry., v.53, p.609-614, 2003.

ZHISHEN, J., MENGCHENG, T. e JIANMING, W. (1999). The determination of flavonoid contents in mulberry and their scavenging effects on superoxide radicals. Food Chemistry, v.64, p.555-559, 1999.

\section{AGRADECIMENTOS}

Os autores agradecem ao $\mathrm{CNPq}$, à Fapemig e à CAPES pelo apoio financeiro na realização da pesquisa. 